\title{
When Behcet's disease meets Stanford type A aortic dissection, heart transplantation is a reliable treatment
}

\author{
Wei Ren ${ }^{1,2 \#}$, Bowen Li ${ }^{1,2 \#}$, Zhiwei Wang ${ }^{1,2}$, Zhiyong Wu ${ }^{1,2}$, Yongle Ruan ${ }^{1,2}$, Jiahui Wang ${ }^{3}$ \\ ${ }^{1}$ Department of Cardiovascular Surgery, ${ }^{2}$ Cardiovascular Surgery Laboratory, ${ }^{3}$ Department of Radiology, Renmin Hospital of Wuhan University, \\ Wuhan 430000, China \\ \#These authors contributed equally to this work. \\ Correspondence to: Zhiwei Wang; Zhiyong Wu. Department of Cardiovascular Surgery, Renmin Hospital of Wuhan University, 238\# Jiefang Road, \\ Wuhan 430000, China. Email: wangzhiwei@whu.edu.cn; wzy2008@whu.edu.cn.
}

\begin{abstract}
Behcet's disease (BD) is an immune system disease characterized by multi-system vascular inflammation. Its occurrence in patients who experience a Stanford type A aortic dissection (AD) is very rare, but extremely dangerous. A 44-year-old male patient was diagnosed with an acute Stanford type A AD and underwent a standard Bentall procedure and total aortic arch replacement plus descending aortic stented elephant trunk implantation. Aortic valve leakage and an aortic root pseudoaneurysm developed 3 months after surgery. At this time, we suspected that this patient had BD. After immunosuppressive treatment, we performed modified Bentall again; however, the heart failure occurred shortly after the second operation. Finally, we successfully treated this patient with a heart transplant. This is the first report of a heart transplant to treat BD with acute Stanford type A AD. In the diagnosis and treatment of acute Stanford type $\mathrm{A} \mathrm{AD}$, in addition to the traditional pathogenic factors, we need to be alert to $\mathrm{BD}$, and heart transplantation may be a good way to treat such patients.
\end{abstract}

Keywords: Behcet's disease (BD); aortic dissection (AD); heart transplantation

Submitted Oct 22, 2019. Accepted for publication Dec 27, 2019.

doi: $10.21037 /$ atm.2020.01.43

View this article at: http://dx.doi.org/10.21037/atm.2020.01.43

\section{Introduction}

Behçet's disease (BD) is a multisystem vascular inflammatory disease of unknown etiology, potentially affecting any organ (1). Vascular involvement has been reported in $7 \%$ to $29 \%$ of BD cases, with mortality of about $20 \%$ in severe cases $(2,3)$, aortic diseases caused by BD often manifested as arteritis and aneurysms, and the lesions can be local or multiple (4). We report a rare case of BD combined with acute Stanford type AAD. We performed three cardiac procedures to treat this patient, including: (I) the Bentall procedure and total aortic arch replacement and stented elephant trunk; (II) modified Bentall procedure; and (III) heart transplantation. At present, 1 year after heart transplantation, the patient is in good condition.

\section{Case presentation}

A 44-year-old Chinese male was transferred to our hospital with a history of 10 hours of severe chest pain. Aortic computed tomography angiography (CTA) indicated Stanford type A AD with aortic root aneurysm (maximum diameter $52 \mathrm{~mm}$ ), which was torn from aortic root to iliac artery without aortic atherosclerosis (Figure 1). Echocardiography showed moderate aortic regurgitation and good left ventricular function (ejection fraction of $60 \%)$. Therefore, the Bentall procedure, total aortic arch replacement and stented elephant trunk was performed under deep hypothermia with circulatory arrest. An aortic root replacement and coronary ostium graft were performed using $25 \mathrm{~mm}$ mechanical Valsalva grafts (St. Jude Medical, 

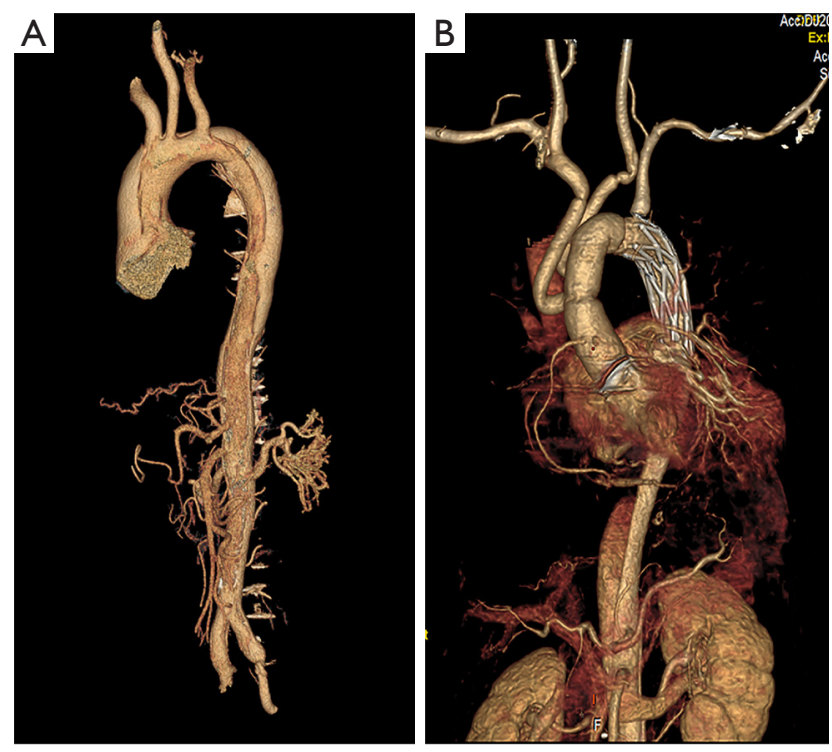

Figure 1 CTA images before and after the first surgery. (A) Aortic computed tomography angiography (CTA) indicated Stanford type A AD with aortic root aneurysm (maximum diameter $52 \mathrm{~mm}$ ), which was torn from aortic root to iliac artery; (B) early in the first postoperative procedure, there was no suggestion for aortic CTA of leakage or pseudoaneurysm formation in the anastomotic site of the aortic root.
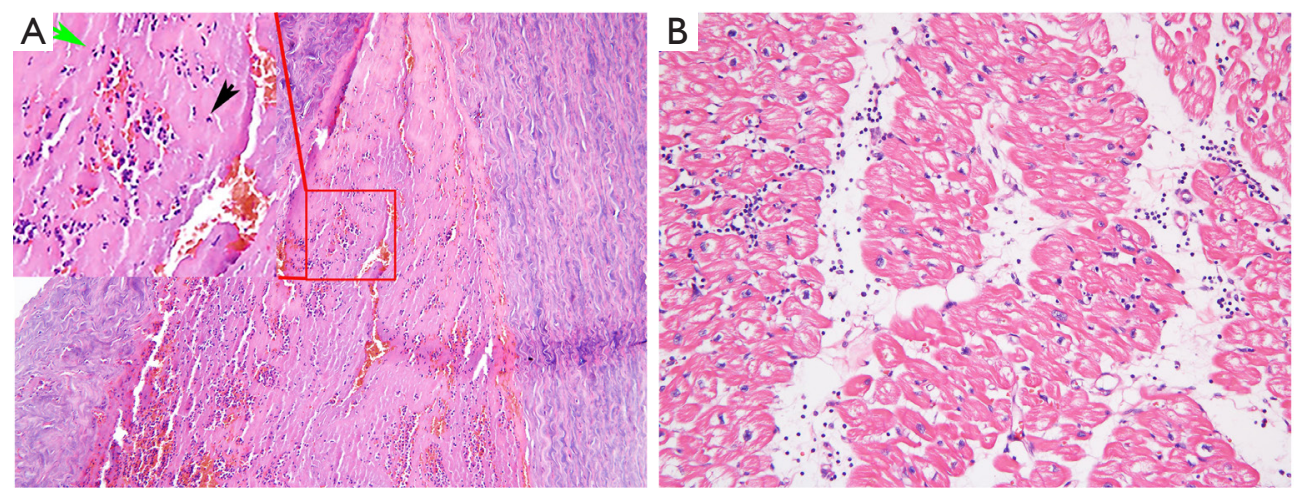

Figure 2 Pathological testing images. (A) HE staining examinations showed intimal fibrosis, middle elastic fiber rupture, and a large number of inflammatory cells, including lymphocytes (green arrow indicates) and neutrophils (black arrow indicates) infiltrating the adventitia of aortic wall; (B) the cardiomyocytes are hypertrophied, and the cells are scattered in lymphocytes $(200 \times)$.

USA). The descending thoracic aorta was implanted with a $26 \mathrm{~mm}$ Cronus aortic stent (MicroPort, China), and the aortic arch was replaced with a Gelweave four-branched vessel (Vascutek Ltd., UK). To reduce bleeding, residual aortic adventitia was wrapped around the aortic root before thoracic closure. Routine pathological examinations showed intimal fibrosis, middle elastic fiber rupture, and a large number of inflammatory cells infiltrating the adventitia of aortic wall (Figure 2). Before discharge, CTA and echocardiography showed no signs of leakage or pseudoaneurysm formation in the anastomotic site of the aortic root (Figure $1 B$ and Figure $S 1 A$ ).

About 2 months later, the patient began to show symptoms of cardiac distress and physical decline. Reexamination with cardiac ultrasonography showed perivalvular leakage in a non-coronary valve, and a pseudoaneurysm could be seen above the aortic root (Figure S1B). CTA identified the formation of a pseudoaneurysm outside the artificial valve of the ascending aorta (Figure $3 A$ ). This is the first case of perivalvular 

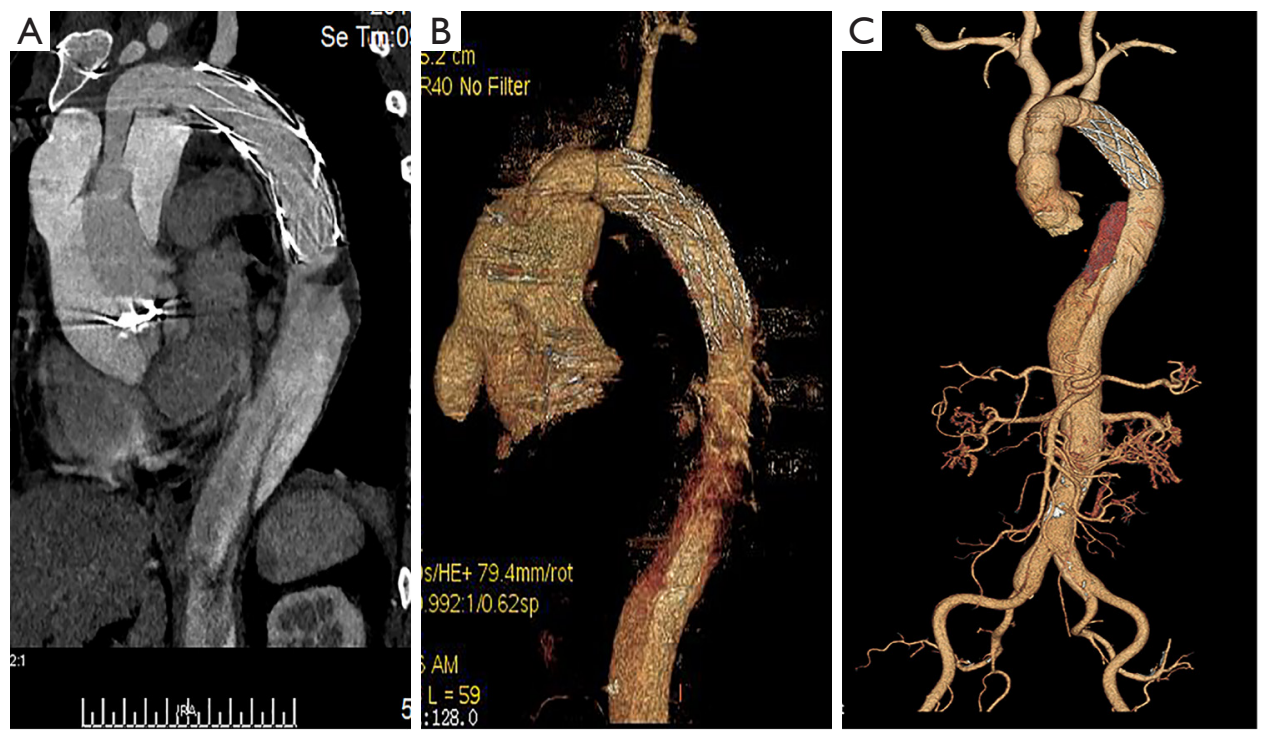

Figure 3 CTA images before and after the heart transplantation. (A) Late after the first procedure, aortic CTA identified the formation of a pseudoaneurysm outside the artificial valve of the ascending aorta; (B) a paravalvular leak occurred again after the second surgery; (C) the aortic root structure is intact after heart transplantation.

leakage after Bentall surgery that our unit has encountered, so we attempted to identify the cause. After reevaluating the patient's medical history, it was found that the patient had no history of hypertension, but experienced more than 3 outbreaks of oral ulcers per year without genital ulcers or eye symptoms, besides, the erythrocyte sedimentation rate (ESR) was $85 \mathrm{~mm} / \mathrm{h}$, and c-reactive protein (CRP) was $27.04 \mathrm{mg} / \mathrm{L}$ during the second admission to hospital, and the acupuncture experiment was positive. After consulting with a rheumatologist, the patient was diagnosed with BD. Preoperatively, prednisone, an oral immunosuppressant, was given at $30 \mathrm{mg} / \mathrm{day}$, and the patient underwent surgery 7 days later after normal level of ESR $(13 \mathrm{~mm} / \mathrm{h})$ and CRP $(6 \mathrm{mg} / \mathrm{L})$ had been achieved. During the second surgery, we strengthened around the annulus with autologous pericardium and sutured the mechanical valve to the left ventricular outflow tract. At the time of discharge, echocardiography showed a diverticulum outside the aortic root, but without blood flow and no perivalvular leakage. The patient did not undergo aortic CTA this time. Prednisone for immunosuppressive therapy was continued after discharge.

Unfortunately, three months after the second surgery, while taking prednisone regularly, the patient complained of chest tightness and pain. Echocardiography and CTA revealed non-coronary valve and right coronary valve leakage (Figure 3B and Figure S1C). Partial blood flow in the cavity of the pseudoaneurysm was being diverted to the right atrium, resulting in increased pulmonary artery pressure. At this time, we performed an orthotopic heart transplantation by bicaval anastomosis. The operation was successful and the patient was considered cured and subsequently discharged. Anatomy of the patient's original heart revealed that the tissues at the anastomotic site of the valve ring and coronary artery had become significantly thickened and friable, with some of the tissues presenting as silt. HE staining suggests hypertrophy of cardiomyocytes, during which lymphocytes infiltrate (Figure 2B). After surgery, immunosuppressive therapy consisting of prednisone, tacrolimus and mycophenolate mofetil was administered, and the level of ESR $(10 \mathrm{~mm} / \mathrm{h})$ and CRP $(5 \mathrm{mg} / \mathrm{L})$ remained normal while blood concentration of tacrolimus was $10 \mathrm{ng} / \mathrm{mL}$. To date, the patient has good cardiac function (LVEF 60\%) (Figure S1D), and no anastomotic leakage was observed by CTA at follow-up 1 year after the third surgery (Figure 3C).

\section{Discussion and conclusions}

BD can cause inflammation of any kind of vessel. Thoracic aortic dissection is a rare and dangerous manifestation of $\mathrm{BD}$, of which acute Stanford type A aortic dissection is the 
most dangerous (5). After a confirmed diagnosis of Stanford type A dissection, emergency surgery is often required. We seldom think of excluding a differential diagnosis of dissection caused by BD, which is made difficult by the non-specific symptoms of BD and the requirement for an extended medical history to diagnose (6). At the same time, acute dissection often induces a systemic inflammatory response and causes elevated inflammatory markers such as ESR and CRP, which also confounds the diagnosis of BD (7). In this case, the patient was at low risk for dissection; he did not present with risk factors such as hypertension, atherosclerosis or Marfan syndrome, so we initially performed a routine dissection repair. However, we were unable to identify the initial cause of the dissection, and BD was suspected only after perivalvular leak occurred. Therefore, for patients without common dissection etiology, a more detailed and retrospective medical history and perform careful physical examination are crucial for successful treatment of the dissection.

The preferred surgical procedure for BD with aortic regurgitation is Bentall surgery, because the valved prosthesis can reduce pressure on the aortic annulus and allows removal of as much inflammatory aortic tissue as possible, greatly reducing the occurrence of perivalvular leakage $(8,9)$. However, our patient developed perivalvular leakage despite undergoing the Bentall procedure; this may be due to activation of the systemic inflammatory response as a result of the acute Stanford type A dissection, leading to severe tissue edema. During the Bentall procedure, the mechanical valve is directly fixed on the inflamed annulus tissue and thus is prone to perivalvular leakage. In addition, Bentall surgery requires grafting of the coronary artery orifice, which is continuous with the aortic wall. During trimming and anastomosis, part of the aortic wall must be sutured, and if BD has already affected the coronary artery orifice, right coronary anastomotic leakage may also occur. Therefore, the key for patients who are both experiencing an acute dissection and in a state of active immune response is mediation of immune system activity followed by surgery once the inflammatory response has subsided.

Patients with Stanford type A dissection are also at risk of the dissection rupturing or poor organ perfusion while waiting for surgery; however, the risks of operating before the immune response has been controlled may be even greater and result in surgical complications and additional subsequent procedures. In the case of our patient, it was extremely difficult to perform the modified Bentall surgery again because of the severe tissue adhesion. Although we used the pericardium to reinforce the annulus, the tissue could not be adequately attached to the prosthesis. We speculate that there was a very small gap between the autologous tissue and artificial prosthesis which expanded after experiencing repeated blood flow, leading to the anastomotic leakage after the second Bentall surgery, despite successful immunosuppression to mediate the inflammatory response.

Heart transplantation solves the problem of multiple anastomoses in the aortic root. In addition, heart transplantation requires immunosuppressive therapy, which also suppresses the inflammation due to BD. Therefore, heart transplantation is a sound choice for patients with perivalvular leaks or root pseudoaneurysms after multiple Bentall surgeries.

The occurrence of $\mathrm{BD}$ concurrent with acute Stanford type A dissection is easily missed due to nonspecific symptoms. Surgery during immune activation potentially increases the risks of paravalvular leaks and pseudoaneurysms. In these cases, it is critical to consult with rheumatologists to control immune activity and the inflammatory response before surgery. Heart transplantation is generally used as the ultimate treatment for endstage heart disease and cannot be performed as a routine operation, but it is an option for patients with recurrent aortic root lesions after standardized immunotherapy. Because this is a single case, the treatment of Stanford type A AD complicated by $\mathrm{BD}$ still requires substantial investigation.

\section{Acknowledgments}

We would like to acknowledge the surgeons, nurses, and clinical staff at the Renmin Hospital of Wuhan University for the high standard of care provided for the patient.

Funding: This work was supported by the National Natural Science Foundation of China (Grant No. 81570428), and Hubei Natural Science Foundation Project (Grant No. 2018CFB141), and Hubei Province Health and Family Planning Scientific Research Project (Grant No. WJ2019M198).

\section{Footnote}

Conflicts of Interest: The authors have no conflicts of interest to declare.

Ethical Statement: The authors are responsible for all aspects of the work to ensure that issues related to the 
accuracy or completeness of any part of the work are properly investigated and resolved. The study was reviewed and approved by Renmin Hospital of Wuhan University. Written informed consent was obtained from the patient for publication of this manuscript and any accompanying images.

Open Access Statement: This is an Open Access article distributed in accordance with the Creative Commons Attribution-NonCommercial-NoDerivs 4.0 International License (CC BY-NC-ND 4.0), which permits the noncommercial replication and distribution of the article with the strict proviso that no changes or edits are made and the original work is properly cited (including links to both the formal publication through the relevant DOI and the license). See: https://creativecommons.org/licenses/by-nc-nd/4.0/.

\section{References}

1. Savey L, Resche-Rigon M, Wechsler B, et al. Ethnicity and association with disease manifestations and mortality in Behçet's disease. Orphanet J Rare Dis 2014;9:42.

2. Farouk H, Zayed HS, El-Chilali K. Cardiac findings in patients with Behçet's disease: Facts and controversies. Anatol J Cardiol 2016;16:529-33.

Cite this article as: Ren W, Li B, Wang Z, Wu Z, Ruan Y, Wang J. When Behcet's disease meets Stanford type A aortic dissection, heart transplantation is a reliable treatment. Ann Transl Med 2020;8(5):251. doi: 10.21037/atm.2020.01.43
3. Mendes D, Correia M, Barbedo M, et al. Behçet's disease-a contemporary review. J Autoimmun 2009;32:178-88.

4. Bouarhroum A, Naouli H, Jiber H. Open surgical repair of abdominal aortic aneurysms in Behçet's disease. J Med Vasc 2019;44:9-18.

5. Kim SW, Lee DY, Kim MD, et al. Outcomes of endovascular treatment for aortic pseudoaneurysm in Behcet's disease. J Vasc Surg 2014;59:608-14.

6. International Team for the Revision of the International Criteria for Behçet's Disease (ITR-ICBD). The International Criteria for Behçet's Disease (ICBD): a collaborative study of 27 countries on the sensitivity and specificity of the new criteria. J Eur Acad Dermatol Venereol 2014;28:338-47.

7. Wen D, Du X, Dong JZ, et al. Value of D-dimer and C reactive protein in predicting inhospital death in acute aortic dissection. Heart 2013;99:1192-7.

8. Chen LW, Wu XJ, Cao H, et al. Valved Conduit Attached to Left Ventricular Outflow Tract for Valve Detachment in Behçet's Disease. Ann Thorac Surg 2017;103:e301-3.

9. Jeong DS, Kim KH, Kim JS, Ahn H. Long-term experience of surgical treatment for aortic regurgitation attributable to Behçet's disease. Ann Thorac Surg 2009;87:1775-82. 

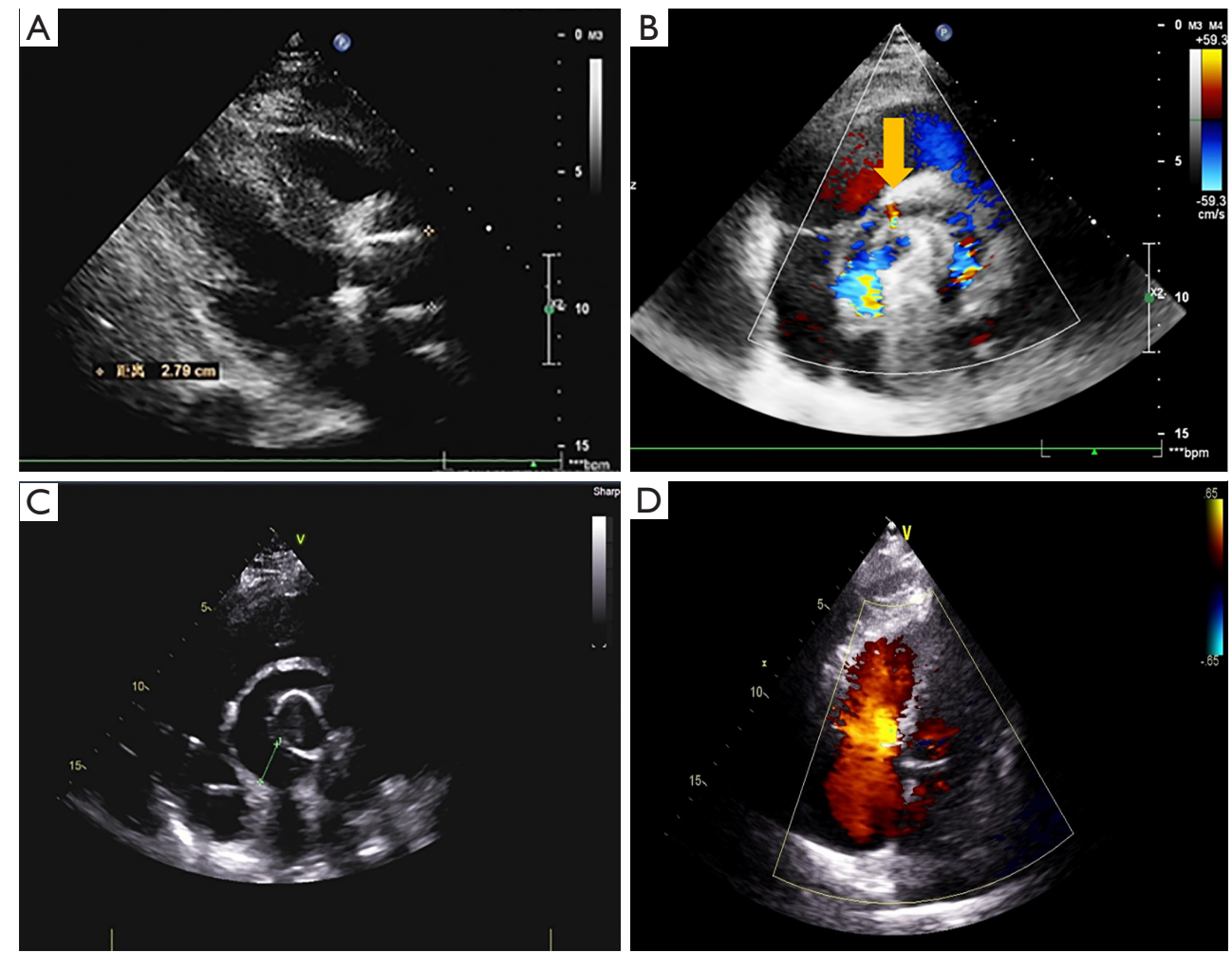

Figure S1 Echocardiographic images of various periods. (A) No paravalvular leaks or pseudoaneurysms were detected early after the first surgery; (B) late after the first procedure, cardiac ultrasonography showed perivalvular leakage in a non-coronary valve, and a pseudoaneurysm could be seen above the aortic root; (C) a paravalvular leak occurred again after the second surgery; (D) there were no abnormalities in cardiac function and aortic root after heart transplantation. 